
parasitóide trichogramma pretiosum em ambientes protegidos. Horticultura Brasileira 27: 080-085.

\title{
Estudo preliminar do controle biológico da traça-do-tomateiro com o parasitóide trichogramma pretiosum em ambientes protegidos
}

\author{
Maria Alice de Medeiros; Geni L Villas Bôas; Nirlene J Vilela; Osmar A Carrijo \\ Embrapa Hortaliças, C. Postal 218, 70359-970 Brasília-DF; maria.alice@embrapa.br; geni@cnph.embrapa.br; \\ nirlene@cnph.embrapa.br
}

\begin{abstract}
RESUMO
O cultivo de tomate em ambiente protegido é economicamente vantajoso para os produtores. No entanto, o ambiente interno da casa de vegetação, quente e seco, favorece o crescimento populacional da traça-do-tomateiro Tuta absoluta (Meyrick) dificultando seu controle, geralmente realizado com inseticidas químicos. O controle biológico da traça-do-tomateiro utilizando o parasitóide Trichogramma pretiosum Riley é indicado como método alternativo aos inseticidas e foi avaliado em ambiente protegido. $\mathrm{O}$ experimento foi conduzido com plantas de tomateiro tutoradas, cv. Larissa, em três modelos de casas de vegetação (arco, capela e convectivo), com 288 plantas em uma área de $160 \mathrm{~m}^{2}$ em cada modelo. Todos os tratamentos foram associados com uma aplicação semanal do inseticida biológico Bacillus thuringiensis e liberações semanais do parasitóide: 1) casa de vegetação com teto convectivo: $200 \mathrm{~cm}^{2} \mathrm{de}$ ovos parasitados em liberação única, ou seja, apenas uma vez por semana; 2) teto em arco: $200 \mathrm{~cm}^{2}$ de ovos parasitados liberados duas vezes por semana, ou seja, $100 \mathrm{~cm}^{2}$ a cada liberação; 3) capela: $400 \mathrm{~cm}^{2}$ de ovos parasitados liberados duas vezes por semana, ou seja, $200 \mathrm{~cm}^{2}$ a cada liberação. A população da traça-do-tomateiro foi amostrada semanalmente, coletando-se 50 folíolos de tomateiro ao acaso, em cada casa de vegetação. Em laboratório, os ovos foram contados, individualizados e mantidos em câmara climatizada para se determinar o número de larvas emergidas e o número de ovos parasitados. A produtividade e o dano causado pela traça foram avaliados em 50 frutos colhidos ao acaso em cada tratamento. A eficiência técnica e econômica do sistema de produção foi analisada em cada modelo de casa de vegetação. Os principais resultados foram: a) as diferentes condições de temperatura e umidade proporcionadas pelas casas de vegetação têm influência sobre o desempenho do parasitóide; b) o uso exclusivo do controle biológico permitiu a produção de tomates por doze semanas em todas as casas de vegetação testadas; c) de acordo com a análise econômica, o tomate produzido em casa de vegetação tipo convectivo apresentou melhor rendimento.
\end{abstract}

Palavras-chave: Tuta absoluta, análise econômica, controle biológico inundativo, parasitóide, tomate.

\begin{abstract}
A preliminar survey on the biological control of South American tomato pinworm with the parasitoid Trichogramma pretiosum in greenhouse models
\end{abstract}

Greenhouse tomato cropping is economically advantageous for the producers. However, the hot and dry environment inside the greenhouse is conducive to tomato pinworm Tuta absoluta (Meyrick) population growth and as a consequence it is difficult to control the pest. Nowadays, the pest is controlled mainly by insecticides. The biological control using the parasitoid Trichogramma pretiosum Riley is pointed out as one of those alternative methods and was evaluated in greenhouse. Experiments were carried out in stacked plants of tomato cv. Larissa, in three greenhouse roof models: arc, chapel and convective, with 288 plants in a $160 \mathrm{~m}^{2}$ area in each type of greenhouse. All treatments were associated with weekly sprays of Bacillus thuringiensis and weekly release of the parasitoid: 1) convective greenhouse: one single release of $200 \mathrm{~cm}^{2}$ of parasitized eggs, once a week; 2) arc roof greenhouse: release of $200 \mathrm{~cm}^{2}$ of parasitized eggs twice a week, $100 \mathrm{~cm}^{2}$ in each release; 3) chapel greenhouse model $400 \mathrm{~cm}^{2}$ of parasitized eggs release twice a week, $200 \mathrm{~cm}^{2}$ in each release. Once a week, 50 leaflets were collected in each greenhouse. The number of eggs/leaf was determined in the laboratory and then the eggs were kept individually in a chamber in order to determine the number of emerged larvae or the number of parasitized eggs. Tomato production and damage to the fruits were determined weekly in 50 tomato plants randomly chosen in each treatment. The technical and economic efficiency of production system were analyzed in the different greenhouse models. The main results were: a) different conditions of temperature and humidity affected parasitoid performance, b) biological control technique provided tomato production for twelve weeks in all greenhouse tested; c) the tomato produced in convective roof greenhouse model provided better income as shown in the economic analysis.

Keywords: Tuta absoluta, economic analysis, inundative biological control, parasitoids, tomato.

\section{(Recebido para publicação em 7 de maio de 2008; aceito em 15 de dezembro de 2008) (Received in May 7, 2008; accepted in December 15,2008)}

$\mathrm{O}$ tomateiro Lycopersicon esculentum (Mill.), de origem peruana, é cultivado em todas as regiões brasileiras, sendo uma das hortaliças mais importantes em área, volume produzido e consumo. A produção do tomate segmenta-se em tomate para processamento industrial e tomate de mesa utilizado para consumo in natura. O tomate é uma hortaliça de elevada im- portância socioeconômica. Além de suas propriedades alimentícias substancialmente benéficas para a saúde humana, a cultura é reconhecida como poderosa fonte geradora de emprego e renda em todos os segmentos de sua cadeia produtiva (Silva \& Giordano, 2000).

A safra de tomate de mesa de 2008 está estimada em 2.358 mil toneladas colhidas em área de 42.038 ha, com pro- dutividade média de 56,1 t/ha conforme levantamento sistemático de produção agrícola (IBGE, 2008). A produção em ambiente protegido é realizada nas regiões Centro-Oeste, Sul e Sudeste e nos estados de Rondônia, Amazonas e Pará. O cultivo de tomateiro em ambiente protegido visa, principalmente, aumentar a produtividade e a qualidade dos frutos, podendo ser implementado em áreas 
pequenas. Esse sistema na região Centro-Oeste é particularmente vantajoso na época da chuva, quando a produção em campo aberto torna-se bastante difícil, devido à ocorrência de doenças. $\mathrm{O}$ período de colheita pode ser prolongado por seis a sete meses, o que proporciona maior produtividade, obtenção de frutos de melhor aparência e qualidade, com maior rentabilidade e compensação do investimento inicial.

A traça-do-tomateiro, Tuta absoluta (Meyrick) (Lepidoptera: Gelechiidae), é uma das principais pragas do tomate no Distrito Federal e região geo-econômica. Esta praga causa sérios danos à tomaticultura e o controle químico não tem sido satisfatório (França, 1993; França et al., 2000; Michereff Filho \& Vilela, 2001; Siqueira et al., 2000). Em ambiente protegido, estudo elaborado por Grande et al. (2003) demonstrou que $84 \%$ dos produtores de hortaliças de Uberlândia (MG) que utilizavam casa de vegetação desistiram da atividade dois anos após seu início. Um dos fatores que geraram este insucesso foi o manejo inadequado de pragas e doenças.

O controle biológico da traça-do-tomateiro com o parasitóide de ovos Trichogramma pretiosum Riley (Hymenoptera: Trichogrammatidae) apresenta bons resultados tanto a campo quanto em casas de vegetação (Parra \& Zucchi, 2004). Medeiros et al. (2006) compararam o controle químico com o controle biológico da traça-do-tomateiro com o T. pretiosum em ambiente protegido. Neste estudo, foi demonstrada a eficiência técnica e econômica do controle biológico da traça-do-tomateiro, por meio da utilização do parasitóide e aplicações do entomopatógeno Bacillus thuringiensis Berliner.

Em ambiente protegido, as condições ambientais diferem das encontradas no campo e podem ter influência sobre o crescimento populacional da traça-dotomateiro e do parasitóide T. pretiosum. O objetivo deste trabalho foi avaliar o controle biológico da traça-do-tomateiro em diferentes sistemas de ambiente protegido.

\section{MATERIAL E MÉTODOS}

Uma unidade de observação foi instalada na Embrapa Hortaliças (DF), de
28/12/00 a 05/04/01, utilizando-se três tipos de casas de vegetação (teto em arco, capela e teto convectivo) (Makishima \& Carrijo, 1998). Foram cultivadas 288 plantas de tomateiro da cultivar Larissa, com $160 \mathrm{~m}^{2}$ de área. O sistema de condução foi o de tutoramento tradicional, com fitilho. T. pretiosum foi criado em Sitotroga cerealella (Olivier) como hospedeiro (Haji et. al., 1998) e as liberações foram iniciadas assim que foram detectados os primeiros adultos da traça-do-tomateiro aos 67 dias após o transplante das mudas. As liberações foram realizadas da seguinte forma: 1) casa de vegetação teto convectivo: $200 \mathrm{~cm}^{2}$ de ovos parasitados em liberação única, ou seja, apenas uma vez por semana; 2 ) teto em arco: $200 \mathrm{~cm}^{2}$ de ovos parasitados liberados duas vezes por semana, ou seja, 100 $\mathrm{cm}^{2}$ a cada liberação; e 3) capela: $400 \mathrm{~cm}^{2}$ de ovos parasitados liberados duas vezes por semana, ou seja, $200 \mathrm{~cm}^{2}$ a cada liberação. Os tratamentos foram associados com uma aplicação semanal do inseticida biológico $B$. thuringiensis (em dose comercial recomendada). As casas de vegetação foram colonizadas naturalmente pela traça-do-tomateiro, de maneira semelhante ao que se verifica em áreas de produção de tomate em ambiente protegido. As populações da traça-do-tomateiro foram avaliadas por meio da coleta semanal de 50 folíolos de tomateiro ao acaso de cada casa de vegetação. Em laboratório, os folíolos foram observados em microscópio estereoscópico para constatar a presença de ovos e larvas da traça. O crescimento populacional da traça foi avaliado baseado no número de ovos por folíolo (até 1,0 ovo/folíolo considerou-se que a população da traça estava sob controle). Os ovos recuperados foram colocados em cápsulas de gelatina e mantidos em câmara climatizada tipo BOD, a $25^{\circ} \mathrm{C}, 70 \%$ UR e $14 \mathrm{~h}$ de fotofase até a emergência do adulto ou do parasitóide. A produtividade e a percentagem de dano causado foram estimados pelo número de frutos produzidos nos tratamentos, desde o início até o final da colheita, considerando-se uma colheita por semana. A temperatura e a umidade interna da casa de vegetação foram monitoradas por meio da utilização de termohigrógrafo.

A receita foi calculada pela quantidade comercial obtida em cada sistema, multiplicada pelo preço de mercado recebido pelo produtor por quilo de tomate vendido. Os custos de produção foram calculados multiplicando-se os coeficientes técnicos pelo preço de mercado dos fatores. Os ovos da traça-do-tomateiro não parasitados foram considerados como perdas, cujo valor foi descontado da receita bruta. Os indicadores técnico-econômicos foram gerados pelo programa de análise econômica por orçamentação parcial (Scolari et al.,1985; FGV, 1997).

\section{RESULTADOS E DISCUSSÃO}

O crescimento populacional da traça-do-tomateiro nos diferentes modelos de casas de vegetação foi semelhante (Figura 1). A população manteve-se baixa até a décima semana (130 dias após o transplante), começando a aumentar a população a partir da $11^{\mathrm{a}}$ semana. $\mathrm{Na} 15^{\mathrm{a}}$ semana (165 dias após o transplante) a população da traça-do-tomateiro atingiu o nível de 1,0 ovo/folíolo na casa de vegetação capela e na semana seguinte nas outras duas casas de vegetação. Este fato demonstra que as medidas de controle biológico empregadas foram eficientes até este período. Em trabalho semelhante, Medeiros et al. (2006) observaram um nível populacional de 7 ovos/folíolo na $15^{\text {a }}$ semana. Em termos quantitativos observou-se que o número de ovos da traça-do-tomateiro foi maior na casa de vegetação capela, seguida pela casa de vegetação teto convectivo e teto em arco.

A taxa de parasitismo por $T$. pretiosum foi de $33 \%$ para a casa teto em arco, $34 \%$ para casa de vegetação capela e $40 \%$ para casa teto convectivo. A casa de vegetação teto convectivo além de ter apresentado maior taxa de parasitismo, foi a que apresentou maior produtividade de tomateiro. No entanto, o tratamento empregado no teto convectivo foi o que usou menor quantidade e freqüência de liberação do parasitóide. As diferentes estruturas proporcionaram variações no ambiente interno, por exemplo, a casa de vegetação teto convectivo apresentou menor temperatura e umidade média, sendo a mais próxima do ambiente externo (Figura 2). Portanto, é possível que as condições 


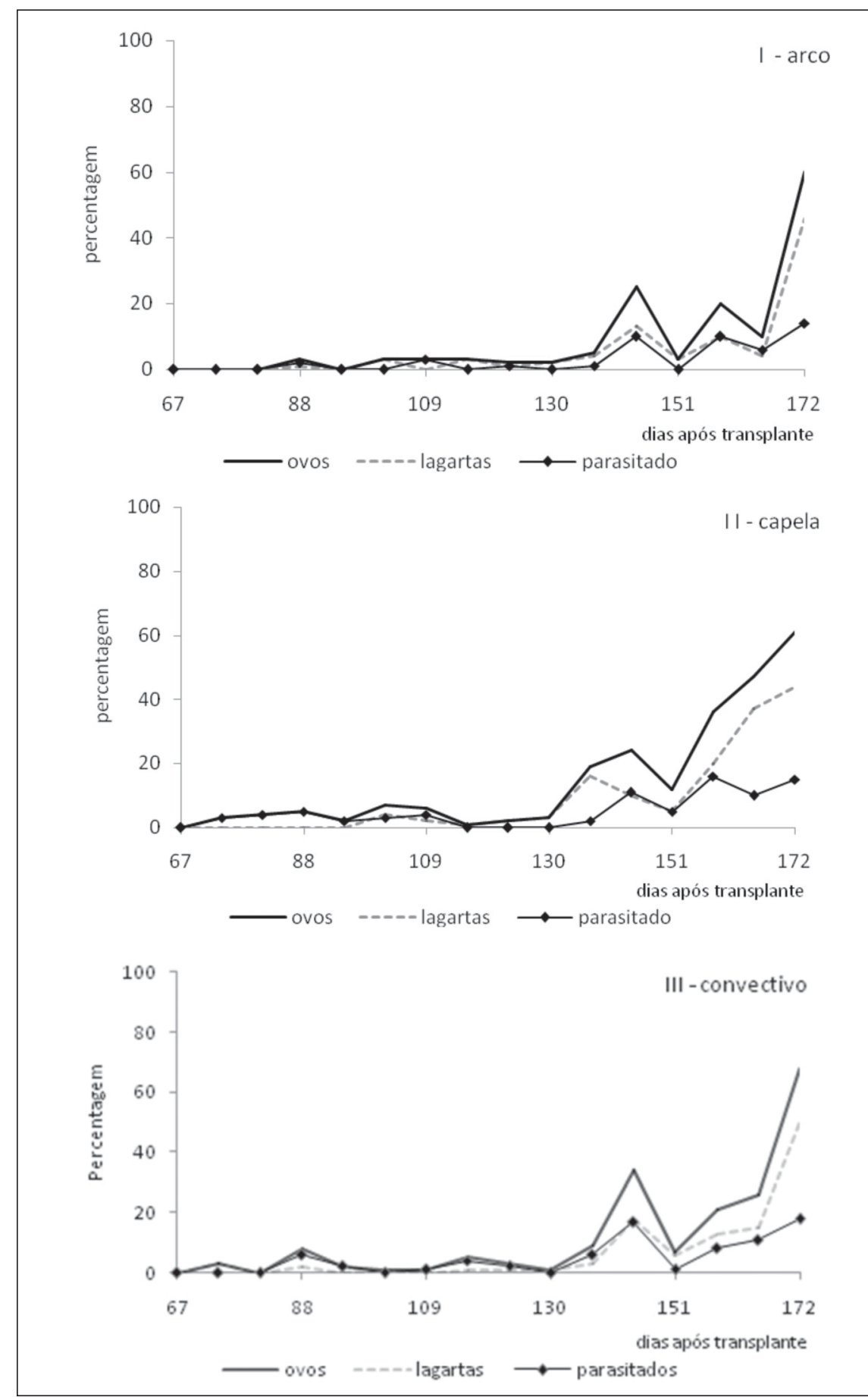

Figura 1. Número de ovos da traça-do-tomateiro coletados em 50 folíolos de tomateiro por semana e o número de lagartas e ovos parasitados por T. pretiosum em tomate cultivado em ambiente protegido. I. Casa de vegetação teto em arco, II. Casa de vegetação capela e III. Casa de vegetação teto convectivo (South American tomato pinworm eggs collected at 50 leaflets/ week, caterpillars and parasited eggs by T. pretiosum in greenhouse tomato crop. I. Arc greenhouse, II. Chapel greenhouse and III. Convective greenhouse). Brasília, Embrapa Hortaliças, 2001.

internas de umidade e temperatura na casa de vegetação tenham influência tanto sobre o crescimento populacional da praga como do parasitóide. De acordo com Pratissoli \& Parra (2000), a viabi- lidade de $T$. pretiosum foi afetada pela variação da temperatura, ou seja, temperaturas de 30 a $32^{\circ} \mathrm{C}$ diminuíram a viabilidade do parasitóide em ovos da traça-do-tomateiro, como também reduzi- ram a duração do ciclo de vida, mas não foi observada interferência da temperatura na razão sexual do parasitóide. Pratissoli et al. (2005) determinaram em condições controladas, em casa de vegetação, que a quantidade de 16 parasitóides/ovo da traça-do-tomateiro proporcionou uma taxa de parasitismo de $80 \%$ e que a partir deste ponto há uma tendência de reduzir a eficiência do parasitóide devido à menor probabilidade de encontrar ovos não parasitados, favorecendo o superparasitismo (Kinipling, 1979). Segundo Sá (1991), o número de parasitóides a ser liberado deve variar em função da densidade do plantio e da intensidade da infestação da praga.

Dentre os fatores físicos a temperatura tem a maior influência nas características biológicas do parasitóide, tais como: fecundidade, duração do ciclo de vida, razão sexual, viabilidade e longevidade (Pratissoli \& Parra, 2001; Noldus, 1989). Trabalhos recentes mostram que existe uma faixa térmica ideal em que o T. pretiosum apresenta melhor desempenho (Pratissoli et al., 2007; Pratissoli et al., 2004; Pereira et al., 2007).

O período de colheita foi de 31/01 até 24/04, ou seja, 12 semanas para todos os tipos de casas de vegetação. A produtividade foi de $2,1 \mathrm{~kg} /$ planta para teto em arco, $1,9 \mathrm{~kg} /$ planta para capela e $2,6 \mathrm{~kg} /$ planta para teto convectivo. Embora com menor quantidade de $T$. pretiosum aplicada, verificou-se que a casa teto convectivo foi a que apresentou maior produtividade e também a que apresentou maior taxa de parasitismo. Esse resultado evidencia que as condições internas da casa de vegetação, tais como temperatura mais amena e umidade mais baixa, proporcionam melhores condições para o desempenho do parasitóide.

Os coeficientes técnicos utilizados na cultura do tomate nos três sistemas de produção desenvolvidos em diferentes tipos de casa de vegetação, apresentaram variações em quantidades somente no item inseticida biológico, sendo que os demais fatores foram utilizados em quantidades iguais em todos os sistemas (Tabela 1). Na casa de vegetação teto em arco e teto convectivo foram aplicados $200 \mathrm{~cm}^{2}$ do parasitóide. 
Tabela 1. Coeficientes técnicos e custos de produção de tomate cultivados com o parasitóide T. pretiosum para controle de traça-do-tomateiro em três tipos de casa de vegetação (technical and economical coefficients for tomato crop system using the parasitoid T. pretiosum to control South American tomato pinworm at three greenhouse models). Brasília, Embrapa Hortaliças, 2001.

\begin{tabular}{|c|c|c|c|c|c|c|}
\hline \multirow{2}{*}{$\begin{array}{l}\text { Tipos } \\
\text { especificações }\end{array}$} & \multicolumn{2}{|c|}{ Arco } & \multicolumn{2}{|c|}{ Capela } & \multicolumn{2}{|c|}{ Convectivo } \\
\hline & Quantidade & Custo (R\$) & Quantidade & Custo (R\$) & Quantidade & Custo (R\$) \\
\hline Sementes CV Larissa (R\$) & 288 & 129,60 & 288 & 129,60 & 288 & 129,60 \\
\hline (Kit fertirrigação) & 1 & 210,00 & 1 & 210,00 & 1 & 210,00 \\
\hline Substrato (Kit) & 1 & 34,00 & 1 & 34,00 & 1 & 34,00 \\
\hline Fungicidas (Kg/lt) & 1 & 87,01 & 1 & 87,01 & 1 & 87,01 \\
\hline Parasitóides $\left(\mathrm{cm}^{2}\right)$ & 66 & 4,62 & 136 & 9,52 & 80 & 5,60 \\
\hline Serviços (R\$) & 10 & 150,00 & 10 & 150,00 & 10 & 150,00 \\
\hline Outros $(R \$)$ & 1 & 250,00 & 1 & 250,00 & 1 & 250,00 \\
\hline Custos operacionais $(R \$)$ & & 865,23 & & 870,13 & & 866,21 \\
\hline
\end{tabular}

Entretanto, a eficiência biológica do parasitóide determinada pelo índice de parasitismo, foi de $33 \%$ e $40 \%$, que gerou o custo de $\mathrm{R} \$ 4,62$ e $\mathrm{R} \$ 5,60$, respectivamente. Na casa de vegetação capela foram aplicados $400 \mathrm{~cm}^{2}$, mas, como o índice de parasitismo foi de $34 \%$, o custo foi de $\mathrm{R} \$ 9,52$. Nas casas de vegetação em referência, os ovos não parasitados por T. pretiosum foram considerados perdas por ineficiência de fatores e descontados da receita bruta, ao preço do fator multiplicado pela quantidade perdida. Dessa forma, o valor das perdas de $T$. pretiosum foram de $\mathrm{R} \$ 9,38$; $\mathrm{R} \$ 18,48$ e $\mathrm{R} \$ 8,40$ nas casas de vegetação teto em arco, capela e teto convectivo, respectivamente (Tabela 2).

A casa de vegetação capela, apesar de ter recebido aplicação de maior quantidade de $T$. pretiosum, apresentou um percentual de $66 \%$ de ovos não parasitados. Neste sistema, a produtividade obtida não apresentou capacidade de sustentar a produção no ponto de equilíbrio, conseqüentemente a eficiência técnico-econômica foi negativa $(-1,90)$. Em outro estudo, Martins et al. (1994) verificaram que a casa de vegetação capela, proporcionou menor produção, quando comparada a outras casas de vegetação. Este fato pode estar relacionado à menor radiação solar incidente neste tipo de casa de vegetação, que reduz a taxa assimilatória, diminuindo conseqüentemente a alocação de reservas para a formação dos frutos. Em virtude de não ter gerado resultados técnico-econômicos desejáveis, o sistema de produção em casa de vegetação tipo capela foi desprezado na análise econômica.

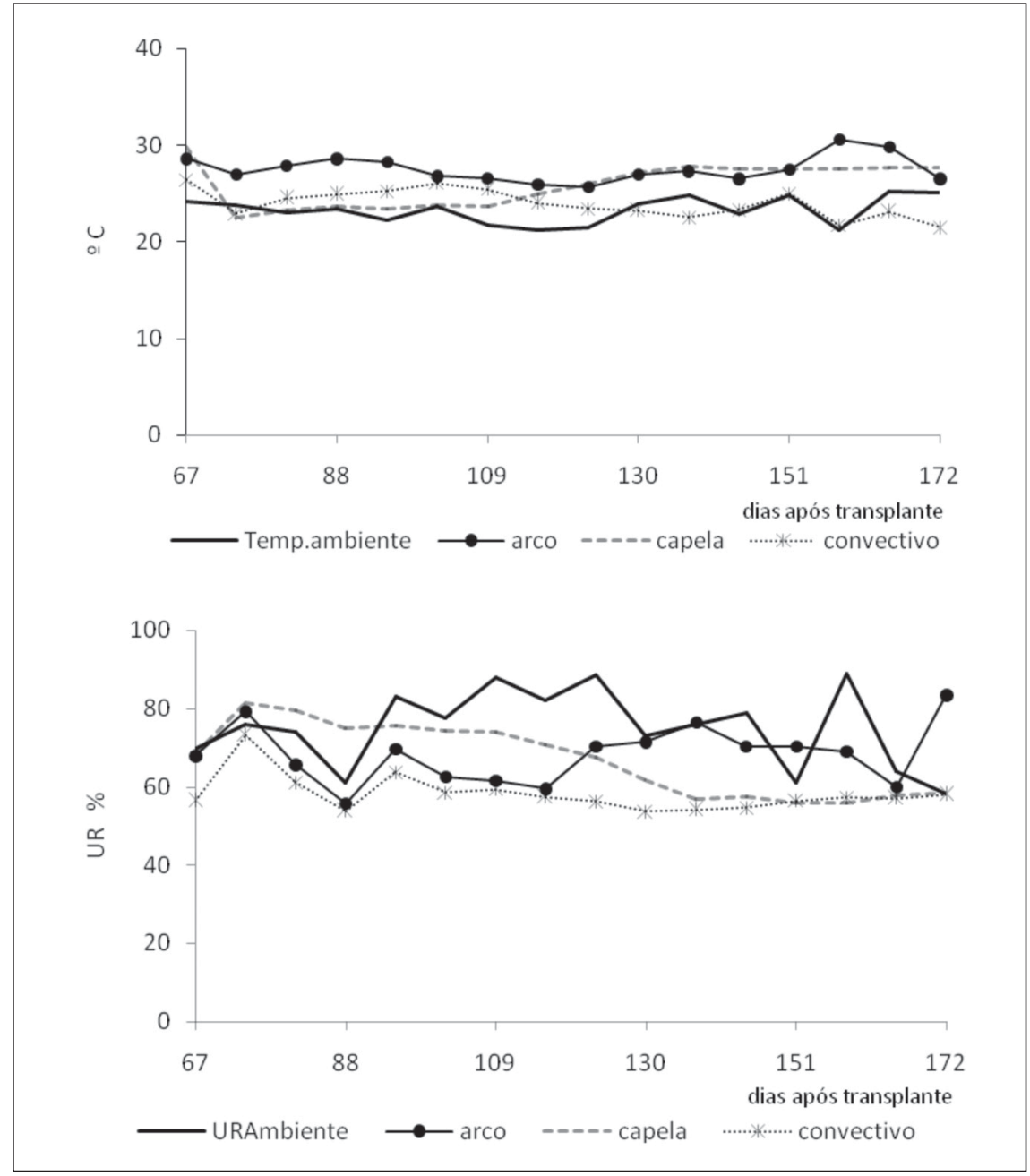

Figura 2. Temperatura e umidade relativa do ar em três tipos de casa de vegetação (emperature and relative humidity air in three greenhouse types). Brasília, Embrapa Hortaliças, 2001.

A maior eficiência técnico econômica foi proporcionada pela casa de vegetação teto convectivo $(34,86)$ (Tabela 2$)$ e a produtividade obtida $(748,80 \mathrm{~kg}) \mathrm{su}-$ perou as produtividades dos demais tratamentos. Os custos operacionais, calculados em $\mathrm{R} \$ 886,21$, foram diluídos pela produtividade em custos unitários de $\mathrm{R} \$ 1,16$. As margens geradas por este sistema foram positivas $(40,51 \%)$, considerando o preço de mercado recebido pelo produtor $(\mathrm{R} \$ 1,63)$. A relação benefício/custo foi favorável $(1,40)$. Adicionalmente, observa-se que em 12 sema- 
Tabela 2. Indicadores técnico-econômicos para sistema de produção de tomate cultivado com o parasitóide T. pretiosum para controle da traça-do-tomateiro em três tipos de casa de vegetação. (technical and economical indicators for tomato crop systems using the parasitoid $T$. pretiosum to control South American tomato pinworm at three greenhouse models). Brasília, Embrapa Hortaliças, 2001.

\begin{tabular}{|c|c|c|c|c|}
\hline Tipos de casas de vegetação & $\operatorname{Arco}(\mathrm{A})$ & Capela (B) & Convectivo (C) & Impacto $\delta \mathrm{C} / \mathrm{A}(\%)$ \\
\hline $\mathrm{A}=$ Produtividade (kg/estufa) & 604,80 & 547,20 & 748,80 & 23,81 \\
\hline $\mathrm{B}=$ Custo operacional $(\mathrm{R} \$)$ & 865,23 & 870,13 & 866,21 & 0,11 \\
\hline$C=B / E=$ Custo unitário $(R \$)$ & 1,43 & 1,59 & 1,16 & $-10,14$ \\
\hline $\mathrm{D}=$ Ineficiência parasitóide $\left(\mathrm{cm}^{2}\right)$ & 9,38 & 18,48 & 8,40 & $-10,45$ \\
\hline $\mathrm{E}=$ Produção Comercial (kg) & 604,80 & 547,20 & 748,80 & 23,81 \\
\hline $\mathrm{F}=$ Preço recebido pelo produtor $/ \mathrm{kg}(\mathrm{R} \$)$ & 1,63 & 1,63 & 1,63 & 0,00 \\
\hline $\mathrm{G}=(\mathrm{F} \times \mathrm{E})=$ Receita Bruta $(\mathrm{R} \$)$ & 985,82 & 891,94 & 1220,54 & 23,81 \\
\hline$H=[(G-B)-D]=$ Receita Líquida & 111,21 & 3,33 & 345,93 & 211,05 \\
\hline I= H/M= Eficiência Econômica dos fatores (UM) & 9,27 & 0,28 & 28,83 & 211,05 \\
\hline$J=(H / B)^{*} 100=$ Eficiência Econômica Sistema $(\%)$ & 12,85 & 0,38 & 39,94 & 210,70 \\
\hline$K=[(G-D) / B]=$ Benefício/custo $(R \$)=$ & 1,13 & 1,00 & 1,40 & 24,00 \\
\hline $\mathrm{L}=[\mathrm{B} /(\mathrm{F}-\mathrm{N})]=$ Ponto de equilíbrio $(\mathrm{kg})$ & 554,63 & 557,78 & 555,26 & 0,11 \\
\hline M = Ciclo de produção e colheita (semanas) & 12,00 & 12,00 & 12,00 & 0,00 \\
\hline $\mathrm{N}=$ Custo unitário do parasitóide $(\mathrm{R} \$)$ & 0,07 & 0,07 & 0,07 & 0,00 \\
\hline$N=[(E-L) / L]^{*} 100=$ Eficiência técnico-econômica $(\%)$ & 9,04 & $-1,90$ & 34,86 & 285,36 \\
\hline
\end{tabular}

nas de colheita, como nos demais sistemas, na casa de vegetação teto convectivo os fatores produtivos apresentaram o melhor desempenho $(28,83 \%)$.

Comparando-se os indicadores técnico-econômicos do sistema de produção desenvolvidos em casa de vegetação teto em arco (A) e teto convectivo (C), observa-se que o sistema de produção " $C$ ” superou o sistema de produção "A" em produtividade $(23,81 \%)$, custos unitários $(-10,14)$, sendo que a ineficiência de parasitóides foi de $(-10,45)$. A eficiência econômica do sistema " $C$ " foi cerca de três vezes maior, resultando que, a cada $\mathrm{R} \$ 1,00$ aplicado neste processo produtivo retornou ao produtor $\mathrm{R} \$ 1,40$ como indicada pela relação benefício/ custo (Tabela 2).

Para alcançar bons resultados empregando o controle biológico da traçado-tomateiro com o parasitóide $T$. pretiosum em ambiente protegido é fundamental considerar que o ambiente interno tem influência sobre populações de insetos. Sob o ponto de vista técnico-econômico concluí-se que a casa de vegetação teto convectivo influenciou favoravelmente a eficiência dos fatores produtivos. Este estudo preliminar indica que os diferentes modelos de casa de vegetação podem influenciar a população de traça-do-tomateiro, como o desempenho de T. pretiosum. É importante que estas informações sejam confir- madas, considerando que modelos mais adequados de casa de vegetação poderão ser propostos, visando promover um manejo mais eficiente de pragas e doenças, de acordo com as variações regionais.

\section{AGRADECIMENTOS}

Ao Dr. Gilmar Paulo Henz pela revisão no manuscrito, aos funcionários Hozanan Pires Chaves e José Gomes Teixeira e aos estagiários que nos auxiliaram durante a condução dos experimentos: Poliana SADiener, Cibila S Simplício e Ramon S Lima, da Universidade Católica de Brasília.

\section{REFERÊNCIAS}

GRANDE L; LUZ JMQ; MELO B; LANA RMQ; CARVALHO JOM. 2003. O cultivo protegido de hortaliças em Uberlândia-MG. Horticultura Brasileira 21: 241-244.

FRANÇA FH. 1993. Por quanto tempo conseguiremos conviver com a traça-dotomateiro? Horticultura Brasileira, 11: 176-178.

FRANÇA FH; VILLAS BÔAS GL; CASTELO BRANCO M; MEDEIROS MA. 2000. Manejo integrado de pragas. In: SILVA JBC; GIORDANO LB (orgs.). Tomate para processamento industrial. Brasília: Embrapa Comunicação para Transferência de Tecnologia/Embrapa Hortaliças. p. 112127.

FGV: Modelo de análise econômica pelo método orçamentação parcial. Rio de Janeiro. FGV. 1997 (Programa computacional).
IBGE. Levantamento sistemático da produção, tomate: produção e área. Rio de Janeiro. Disponível em: http:www.sidra.ibge. gov.br. Acesso em outubro de 2008.

KINIPLING EF. 1979. Insect population suppression by augmenting the number of parasites and predators in crop ecosystems. In: The basic principles of insect populations suppression and management. Washington: USDA. p.135-197. (Agriculture Handbook, 512).

HAJI FNP; VELASQUEZ JJ; BLEICHER E; ALENCAR JA: HAJI AT; DINIZ RS. 1998. Tecnologia de produção massal de Trichogramma spp. Petrolina, PE: Embrapa-CPATSA, 24p.

MAKISHIMA NO; CARRIJO OA. 1998. Cultivo protegido do tomateiro. Brasília: EMBRAPA-CNPH. 20p. Embrapa Hortaliças. Circular Técnica, 13.

MARTINS G; CASTELLANE PD; VOLPE CA. 1994. Influência da casa de vegetação nos aspectos climáticos e em época de verão chuvoso. Horticultura Brasileira, 12: 131-135.

MEDEIROS MA; JUNQUEIRA NV; FRANÇA FH. 2006. Eficiência técnica e econômica do controle biológico da traça-do-tomateiro em ambiente protegido. Horticultura Brasileira 24: 180-184.

MICHEREFF FILHO M; VILELA EF. 2001. Traça-do-tomateiro, Tuta absoluta (Lepidoptera: Gelechiidae). In: VILELA EF, ZUCCHI RA, CANTOR F. (eds.) Histórico e impacto das pragas introduzidas no Brasil. Ribeirão Preto: Holos. p.81-84.

NOLDUS LPJJ. 1989. Semiochemicals, foraging behaviour and quality of entomophagus insects for biological control. Journal of Applied Entomology 108: 425-451.

PARRA JRP; ZUCCHI RA. 2004. Trichogramma in Brazil: feasibility of use after twenty years of research, Neotropical Entomology 33: 271-281. 
PEREIRA FF; BARROS R; PRATISSOLI D; PEREIRA CLT; VIANNA UR; ZANUNCIO JC. 2007. Capacidade de parasitismo de Trichogramma exiguum Pinto \& Platner, 1978 (Hymenoptera: Trichogrammatidae) em ovos de Plutella xylostella (L., 1758) (Lepidoptera: Plutellidae) em diferentes temperaturas. Ciência Rural 37: 297-303.

PRATISSOLI D; PARRA JRP. 2000. Desenvolvimento e exigências térmicas de Trichogramma pretiosum Riley, criados em duas traças do tomateiro. Pesquisa Agropecuária Brasileira 35: 1281-1288.

PRATISSOLI D; PARRA JRP. 2001. Seleção de linhagens de Trichogramma pretiosum para o controle das traças Tuta absoluta e Phthorimaea operculella. Neotropical Entomology 30: 1-6.

PRATISSOLI D; PEREIRA FF; BARROS R; PARRA, JRP; PEREIRA CLT. 2004. Parasitismo de Trichogramma pretiosum em ovos da traça-das-crucíferas sob diferentes temperaturas. Horticultura Brasileira 22: 754-757.
PRATISSOLI D; POLANCZYK RA; PEREIRA CL; FURTADO ISA; COCHETO JG. 2007. Influência da fase embrionária dos ovos da traça-das-crucíferas sobre fêmeas de Trichogramma pretiosum com diferentes idades. Horticultura Brasileira 25: 286-290.

PRATISSOLI D; THULER RTI; ANDRADE GS; ZANOTTI LCM, SILVA AF. 2005. Estimativa de Trichogramma pretiosum para o controle de Tuta absoluta em tomateiro estaqueado. Pesquisa Agropecuária Brasileira 40: 715-718.

SÁ LAN. 1991. Bioecologia de Trichogramma pretiosum Riley, 1879, visando avaliar o seu potencial para o controle de Spodoptera frugiperda (Smith, 1797) $e$ Helicoverpa zea (Boddie, 1850) em milho. Piracacaba: USP ESALQ. 170p. (Tese doutorado).
SCOLARI DG; COSTA MEFA; SOUZA MC. 1985. Programa de análise econômica através de orçamentação parcial (ANECOR). Planaltina: EMBRAPA-CPAC. 43p. (EMBRAPA-CPAC. Documentos, 13).

SIQUEIRA HA; GUEDES RNC; PICANÇO M. 2000. Insecticide resistance in populations of Tuta absoluta (Lepidoptera: Gelechiidae). Agricultural and Forest Entomology, 2: 147-153.

SILVA JBC; GIORDANO LB. 2000. Tomate para processamento industrial. Brasília: Embrapa Comunicação para Transferência de Tecnologia/Embrapa Hortaliças. 168p 